\title{
ESTUDOS SOBRE A EPIDEMIOLOGIA DA DOENÇA DE CHAGAS NO CEARÃ. XXII - ECOLOGIA DE TRIATOMINEOS EM PEREIRO *
}

\author{
Joaquim Eduardo de Alencar, Otávio Ferreira Bezerra e \\ Oswaldo Freire de Faria Filho
}

\begin{abstract}
Foi escolhida, para demolição, uma casa no municipio de Pereiro, território de prevalência de Triatoma, pseudo maculata, onde raros exemplares de Triatoma, braziliensis são encontrados. Numa área de 13 casas, onde vivem 74 pessoas, foram capturados $41 \mathrm{~T}$., pseudo maculata que apresentaram taxas de infecção de 2,4\%. Em Pereiro a infecção humana é de 2,2\% e é baixa a infecção de animais: $2,0 \%$ pelo xenodiagnóstico. A casa selecionada foi desinsetizada mais de um ano antes da demolição e 20 dias antes da demolição uma captura mostrou 20 T., pseudo maculata, sendo 50\% infectados. Durante a domolição estavam presentes: 6 pessoas, 1 cão, 1 gato, 1 porco e 30 galinhas; na ocasião foram capturados 221 triatomíneos, no teto $\left(0,5\right.$ por $\left.\mathrm{m}^{2}\right)$; e nas paredes internas e externas $\left(14\right.$ por $\left.m^{2}\right) ; 180$ exemplares foram examinados e apresentaram 7,2\% de infecção. Concluiu-se que os métodos normais de captura mostram pequena parte da colônia de triatomineos numa casa habitada e a taxa de infeçãa do T., pseudomaculata observada revela sua capacidade de transmissão do Trypanossoma cruzi, pois $20 \%$ dos exemplares infectados foram encontrados no quarto.
\end{abstract}

\section{INTRODUÇÃO}

O estudo da epidemiologia da doença de Chagas vem sendo enriquecido pela introdução de métodos de observação cada vez mais sofisticados. No que diz respeito à colonização de casas por triatomíneos domésticos ou semidomésticos, tem sido dificil determinar o número real de exemplares existentes num determinado momento em habitação humana, o que revelaria - grau de risco a que estariam submetidos os seus moradores $^{1}$. Para que se pudesse obter essa informação, buscou-se avaliar a relação entre o número de exemplares observados por captura na casa, utilizando-se piriza como desalojante, e o obtido por demolição da casa após a captura.

Outra informação de importäncia que se busca obter é a prevalência das diversas espécies de triatomíneos, domésticos ou semidomésticos, em habitações borrifadas com inseticidas clorados e o tempo de recolonização dessa habitação. Não sabemos ainda os ecótopos onde o triatomineo resiste.
Alguns projetos de pesquisa têm sido apresentados, sem lograr o mecenas respectivo; por isso os autores intentaram observações limitadas, selecionando para isso áreas de prevalência exćlusiva ou a maior das espécies vetoras dé Trypanosoma cruzi no Ceará.

O município Pereiro é uma área de maior prevalência do $T$. pseudomaculata no Ceará ${ }^{2}$.

\section{MATERIAL E MÉTODO}

Numa área de estudos sobre a epidemiologia da doença de Chagas no municipio de Pereiro, foi selecionado um agrupamento de 17 casas, onde vivem 98 pessoas, no sítio S. Gonçalo. Realizada uma captura preliminar, revelou-se a existência de uma predominância de $T$. pseudomaculata.

Foi feita a indagação sobre a concordância do proprientário de algumas das casas infectadas no sentido de demoli-las, após indenização dos gastos para reconstrução. Verificada a data da última borrifação e estabelecido o prazo da demolição da casa, a qual foi realizada 490 dias depois dessa última borrifação; sendo, por isso,

\footnotetext{
* Trabalho do Centro de Ciências da Saúde da Universidade Federal do Ceará e da SUCAM, realizado
} com auxilio do CNPq (PDE - 02-1-04). 
catalogada como não borrifada.

Foi realizada uma captura preliminar, com a duração de 20 minutos, e logo após foi realizada a demolição da casa, depois de medidas as paredes e recenseados os habitantes e animais.

A demolição se fez metro por metro, começando pelas paredes e terminando pelo teto; cada torrão de barro era desfeito e examinado para verificar a existência de insetos e em especial triatomíneos, que eram colocados em vidros rotulados com a indicação do local.

Todos os triatomíneos foram examinados para classificação e verificação de infecção pelo T. cruzi.

\section{RESULTADOS}

Censo - Na localidade de S. Gonçalo, em 29 de novembro de 1977 existiam 74 habitantes em 13 casas, o que equivale a 5,69 habitantes em cada casa (Tabela n? 1). Durante 20 minutos foi realizada captura de triatomíneos, encontrandose $41 T$. pseudomaculata, sendo 1 infectado com $T$. cruzi $(2,4 \%)$.

$\mathrm{Na}$ casa n. 15 , selecionada para demolição, residem 6 pessoas e 2 animais, dos quais foram feitos xenos. A captura prévia, feita a 09.11.77. revelou somente $T$. pseudomaculata $(100 \%) \mathrm{e}$ nenhum $T$. braziliensis com uma taxa de infecção global de $5 \%$ (Tabela n? 2).

\section{Tabela I}

Estudos sobre a epidemiologia da doença de Chagas no Ceará.

Municipio de Pereiro - Localidade: São Gonçalo - 1977.

Caracteristicas das Casas

\begin{tabular}{|c|c|c|c|c|c|c|c|c|c|c|c|}
\hline \multirow{3}{*}{\multicolumn{2}{|c|}{$\begin{array}{l}\text { No de } \\
\text { Casas }\end{array}$}} & \multicolumn{4}{|c|}{ PAREDE } & \multicolumn{2}{|c|}{ TE TO } & \multicolumn{3}{|c|}{ PISO } & \multirow{3}{*}{$\begin{array}{l}\text { Altitude } \\
\text { Média } \\
\text { (M) }\end{array}$} \\
\hline & & \multicolumn{2}{|c|}{ Barro } & \multicolumn{2}{|c|}{ Tijolo } & \multirow{2}{*}{ Telha } & \multirow{2}{*}{ Palha } & \multirow{2}{*}{ Barro } & \multirow{2}{*}{ Cimento } & \multirow{2}{*}{ Tijolo } & \\
\hline & & C/Reb. & S/Reb. & C/Reb. & S/Reb. & & & & & & \\
\hline & 13 & - & 12 & - & 1 & 13 & - & 13 & - & - & 669 \\
\hline$\%$ & 100,0 & - & 92,3 & - & 7,7 & 100,0 & - & 100,0 & - & - & \\
\hline
\end{tabular}

Características da casa na demolição - A demoliçõa foi realizada nos dias 29 e 30 de novembro de 1977. A área do piso era de 30 metros quadrados; a casa era habitada por 6 pessoas, o que corresponde a $5 \mathrm{~m}^{2}$ por habintante. As paredes internas somaram $30 \mathrm{~m}^{2}$, as paredes externas $41 \mathrm{~m}^{2} \mathrm{e}$ a área do teto $39 \mathrm{~m}^{2}$.

Triatomíneos capturados na demolição (Tabela 3)

a) no teto $\ldots \ldots \ldots 16(0,4)$ por $\mathrm{m}^{2}$;

b) nas paredes internas . . $164(5,5)$ por $\mathrm{m}^{2}$;

c) nas paredes externas . 0

d) total de capturados, incluindo os não examinados ............. 221

Razões observadas:

a) triatomíneos por $\mathrm{m}^{2}$ de área . . . 2,76

b) triatomíneos por aposento . . . .74,00

c) triatomíneos por habitante . . . 36,83

d) triatomineos por cão ou gato . . . 110,50

e) triatomíneos por cão, gato ou ave. . . .6,91

Infeccão pelo $T$. cruzi e fases evolutivas (Tabelas 3 e 4 )

a) No de adultos ... . . 36 (4 infectados) b) NO de ninfas . . . . . . 144 (9 infectados)

c) No total de triatomíneos . $180(13$ infectados $)(7,2 \%) \quad \operatorname{tados})(7,2 \%)$

Ecótopos mais habitados:

a) Sala . . . . . 100 (7 infectados) $-(7,0 \%)$

b) Quarto . . . . . 22 (3 infectados) - 13,6\%)

c) Cozinha. . . . . 42 (2 infectados) $-(4,8 \%)$

d) Teto . . . . . . 16 (1 infectados $)-(\ldots)$

\section{DISCUSSÃO}

Densidade da colônia - 0 T. Pseudomaculata tem características que o colocam como habitante de ecótopos artificiais peridomiciliares $^{2}$. Nessa observação no entanto ele aparece em quantidades mais elevadas que habitualmente, pela demolição da casa; antes da demolicão a captura prévia revelou apenas um décimo do número observado na demolição. .

Ecótopos mais habitados e taxa de infecção As paredes internas são as mais habitadas, sendo a taxa de infecção mais elevada. Se tivermos em conta o cômodo da habitação, veremos que o maior número de exemplares foi observado na 
Tabela II

Pereiro - São Gonçalo

Triatomíneos examinados na casa n: 15

(Demolida) - 1977

\begin{tabular}{cllcc}
\hline \multirow{2}{*}{$\begin{array}{c}\text { No de Capturas } \\
\text { e Datas }\end{array}$} & Ecótopos & \multicolumn{3}{c}{ Triatomíneos Capturados } \\
\cline { 3 - 5 } & & Ninfas & Adultos & T o t a l \\
\hline 1. 26/01/1976 & Negativa & - & - & - \\
\hline 2. $19 / 08 / 1977$ & Sala & 05 & 03 & $08(-)$ \\
\hline 3. 09/11/1977 & Sala & $17(1)$ & $01(-)$ & $18(1)$ \\
& Quarto & $-(-)$ & $02(-)$ & $02(-)$ \\
\hline 4. 29/11/1977 & Sala & $02(-)$ & $02(-)$ & $04(-)$ \\
& Quarto & $04(-)$ & - & $04(-)$ \\
& Cozinha & $05(-)$ & - & $05(-)$ \\
\hline 5. 29/11/1977 & Sala & $78(5)$ & $22(2)$ & $100(7)$ \\
& Quarto & $16(2)$ & $06(1)$ & $22(3)$ \\
Demolição & Cozinha & $37(1)$ & $05(1)$ & $42(2)$ \\
& Teto & $03(-)$ & $13(1)$ & $16(1)$ \\
\hline T O T A L & & $167(9)$ & $54(5)$ & $221(14) 6,3 \%$ \\
\hline
\end{tabular}

NOTA: Entre parênteses - os positivos

Tabela III

Estudos sobre a epidemioloiga da doença de Chagas no Ceará.

Triatomineos capturados: Distribuição por ecótopos e fases evolutivas

São Gonçalo - Município de Pereiro - 29/30 - Novembro de 1977

\begin{tabular}{|c|c|c|c|c|c|c|c|c|c|c|c|c|}
\hline \multirow{3}{*}{$\begin{array}{l}\text { Fases } \\
\text { Evolutivas }\end{array}$} & \multicolumn{12}{|c|}{ TRIATOMINNEOS CAPTURADOS E SEUS ECÓTOPOS } \\
\hline & \multicolumn{2}{|c|}{ Sala } & \multicolumn{2}{|c|}{ Quarto } & \multicolumn{2}{|c|}{ Cozinha } & \multicolumn{2}{|c|}{ Teto } & \multicolumn{4}{|c|}{ Total } \\
\hline & Exam. & Pos. & Exam. & Pos. & Exam. & Pos. & Exam. & Pos. & Capt. & Exam. & Pos. & $\%$ \\
\hline Ninfa de $1 ?$ & 09 & - & - & - & - & - & 01 & - & 21 & 10 & - & - \\
\hline Ninfa de 11 ? & 09 & 02 & - & - & 05 & - & 03 & - & 25 & 17 & 02 & - \\
\hline Ninfa de Ill? & 21 & 03 & 09 & - & 11 & 01 & 05 & 01 & 59 & 46 & 05 & 10,9 \\
\hline Ninfa de IV? & 19 & - & - & - & 11 & - & 02 & - & 38 & 32 & - & - \\
\hline Ninfa de $V ?$ & 20 & - & 07 & 02 & 10 & - & 02 & - & 40 & 39 & 02 & 5,1 \\
\hline Macho & 08 & - & 02 & - & 03 & 01 & - & - & 14 & 13 & 01 & - \\
\hline Fêmea & 14 & 02 & 04 & 01 & 02 & - & 03 & - & 24 & 23 & 03 & 13,0 \\
\hline TOTAL & 100 & 07 & 22 & 03 & 42 & 02 & 16 & 01 & 221 & 180 & 13 & 7,2 \\
\hline
\end{tabular}


sala, porém a maior taxa de infecção observouse no quarto. Devemos ver numa casa de 3 cômodos com 6 pessoas que tanto a sala como a cozinha servem de dormitório e aí também os triatomíneos estavam infectados. As taxas de infecção contrastam mesmo com a taxa média de infecção observada no Estado do Ceará ${ }^{2}$.

O número de exemplares observado no teto não foi elevado e aí somente um estava infectado. Deve-se a isso, ao que nos parece, o demorado lapso de tempo que a casa passou sem borrifaçãó (490 dias) permitindo assim adequadas condições de vida do triatomíneo nas paredes da habitação. O teto parece constituir um refú gio de triatomíneo quando a desinsetização é feita somente nas paredes.

As taxas de infecção, tendo em conta a razão triatomíneo habitante, podem ser consideradas como suficientes para a realização da transmissão do $T$. cruzi aos habitantes da casa e dos animais domésticos existentes: 1 cão e 1 gato. Entretanto nenhum dos habitantes e nenhum dos animais revelou estar infectado pelos métodos utilizados: teste de imunofluorescência indireta para as pes soas e xeno para o cão e o gato.

Tabela IV

Estudos sobre a epidemioloiga da doença de Chagas no Ceará.

Animais capturados na casa no 15 , por ocasião da demolição (29/30.XI.1977)

São Gonçalo - Município de Pereiro

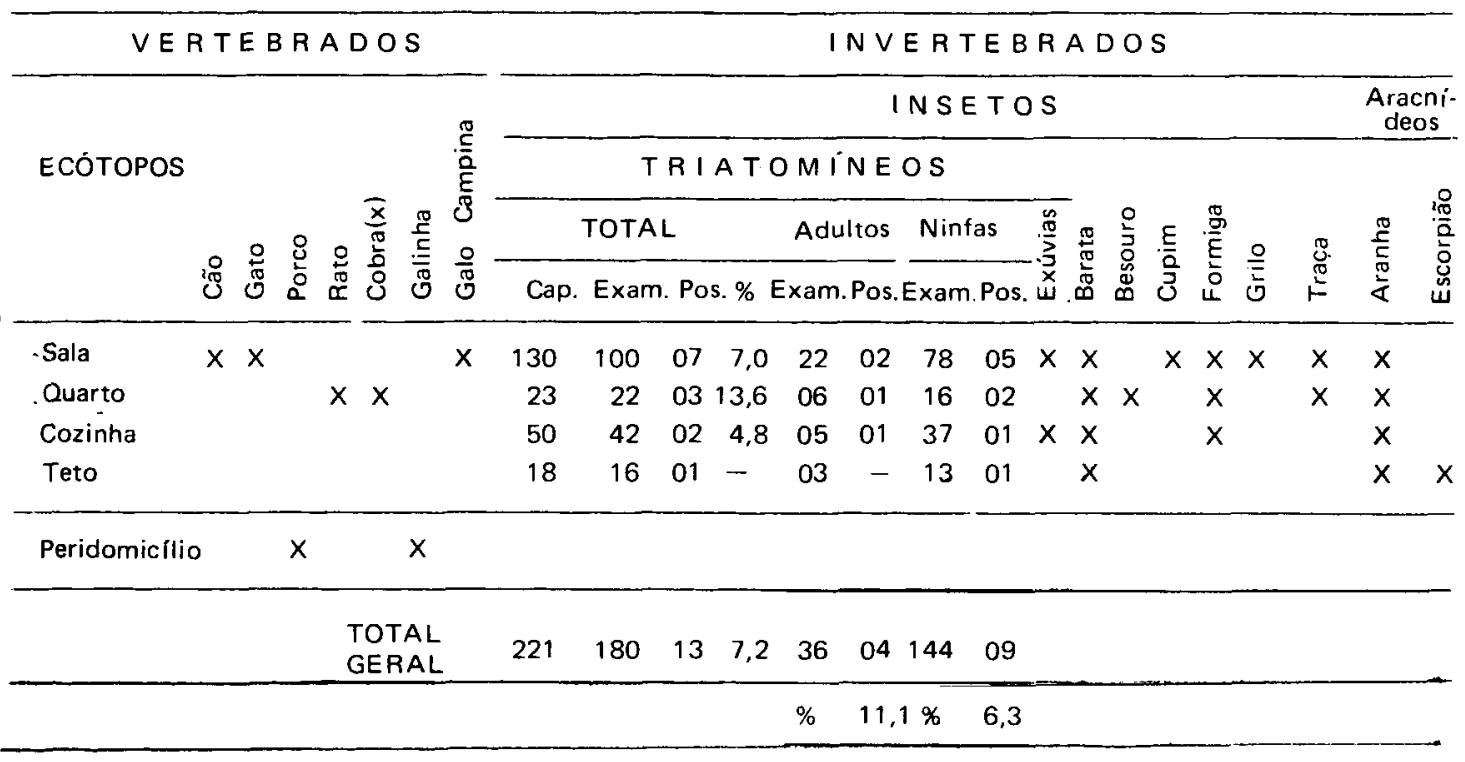

\section{CONCLUSÃO}

As informações obtidas na pesquisa relatada permitem concluir:

1. Os métodos normais de captura de triatomineos numa casa habitada mostram apenas uma pequena parte da colônia;

2. $\mathrm{Na}$ área, a prevalência quase exclusiva de T. pseudo maculata permitiu-lhe ocupar ecóto pos artificiais intradomiciliares, o que não é o habitual da espécie;

3. Ficou evidente a capacidade de transmissão do $T$. cruzi pela espécie, visto que a taxa de infecção era suficiente e a maior parte dos exemplares infectados foi observada no quarto.

\section{SUMMARY}

In the municipality of Pereiro an area where Triatoma pseudomaculata is prevalent and Tria. toma braziliensis only rarely seen, a house was selected for demolition. It was one of 13 houses of a community of 74 people. In a survey 41 T. pseudomaculata were captured only $2.4 \%$ of which were infected with Trypanosoma cruzi. Human infection in the area was $2.2 \%$ and there was a low infection rate in domestic animals $(2.0 \%)$.

The house to be demolished was sprayed with BHC one year previously. $20 \mathrm{~T}$. pseud smaculata captured before demolition had a $50 \%$ infection 
rate. At demolition 6 people, 1 dog, 1 cat, 1 pig and 30 chickens were present in the house. 221 triatomids were captured at demolition, 0.5 per square metre in the roof and 1,4 per square metre in internal and external walls. 180 were examined and $7.2 \%$ were infected.

We conclude that by normal methods only a small proportion of the bug population is captured in a house. The infection rate in T. pseudomaculata reveals its capacity for transmitting $T$. cruzi especially as $20 \%$ of the infected bugs were captured in te bedroom.

\section{REFERÉNCIAS BIBLIOGRÁFICAS}

1. MINTER, D. M. - The Chagas' Disease Research Unit. Relatório datilografado. Salvador Bahia. 13 ${ }^{\text {a }}$ Reunião do Comitê Consultivo sobre Pesquisa Médica. Pan American Health Organization, jun., 1974

2. ALENCAR, J. E., SANTOS, A. R.,

BEZERRA, O.F. \& SARAIVA, T. M. Distribuição geográfica dos principais vetores de endemias no Estado do Ceará. I Triatomíneos. Rev. Soc. Bras. Med. Trop., 10 (5):261-284, 1976. 